\title{
Lost in Translation: Can We Afford to Miss the Trees for the Forest?
}

\author{
Stephen R. Hammes • Carol A. Lange
}

Published online: 1 July 2014

(C) Springer Science+Business Media New York 2014

Is there a favorite Ivy League school to which you would like to apply? If so, the odds of an outstanding high school student getting accepted into one of these top-tier institutions averages out to approximately $9 \%$. Put another way, if you are one of the best and brightest in your high school, you have about a 1:10 chance of getting into the Ivy League school of your choice. These are pretty low odds. Then again, this is the Ivy League, considered by many to be the best of the best. Furthermore, regardless of whether you are accepted, is your future really "blown" if you are not accepted to your Ivy of choice? Of course not! There are dozens if not hundreds of amazing colleges in the USA with very favorable acceptance rates. For example, UC Berkeley, The University of Chicago, Duke University, and Georgetown University each accept approximately $17 \%$ of applicants. The University of Rochester, New York University, and The Universities of Minnesota, Virginia, Michigan, and Texas all have acceptance rates of over $30 \%$. Thus, any smart successful high school student will find a fantastic place to start their academic career. Furthermore, if a student continues to be successful at any one of these colleges, then graduate or medical school is easily reachable.

Let us now compare these numbers to those of an established successful scientist trying to stay afloat. Currently, the success rate for an NIH R01 grant is the same as the chances of a top high school student getting into his/her Ivy League School of choice - about $10 \%$. Again, these are pretty low odds. Unfortunately, unlike the top high school student, who has a myriad of other viable options, if this successful

S. R. Hammes

University of Rochester School of Medicine and Dentistry,

Rochester, NY 14642, USA

C. A. Lange $(\bowtie)$

University of Minnesota, Minneapolis, MN 55455, USA

e-mail: lange047@umn.edu scientist cannot maintain R01 funding, there are few alternatives - a lifetime of work will be tossed aside, with jobs and careers damaged if not lost. These are sobering times. As reviewers try to pick and choose between outstanding proposals, successful careers hang in the balance. Which research proposals should be selected for potential funding and which should be left in the pile? A major criterion for making these critical decisions revolves around judgment of the work as "innovative" and "impactful" with high "translational" potential. Yet is anybody really capable of making this judgment? Should we even be trying to do so? Examples from history would argue that the answer is emphatically "NO!"

The decade was the 1950s. Microbiologist Salvador Luria, when looking at his petri dishes, discovered that some bacterial hosts were able to suppress bacteriophage growth, while others were not. For lack of a better term, Dr. Luria called these suppressing bacteria "restricting" hosts [1]. This observation sounds interesting but pretty tame. Not clearly innovative and definitely not at all translational. Nearly 10 years later, another microbiologist, Werner Arber, discovered that these restricting bacteria actually produced an enzyme that cleaves phage DNA and destroys it [1]. To be consistent with Luria's terminology, this enzyme was deemed a "restriction enzyme." Finally, after about 10 more years, microbiologist Hamilton O. Smith discovered a second enzyme in another restricting bacteria (Hemophilus influenza) that cleaved DNA at a very specific DNA sequence [2]. This enzyme was called "Hind." Now, the story is becoming a little more interesting, but still is significant only in the world of bacterial biology — not overly innovative and still with very limited translational potential. In fact, this series of observations, which spanned nearly 20 years, would very likely have failed to meet current NIH definitions of translational because nobody could really predict the true potential of their findings until Daniel Nathans put all of these findings together and demonstrated how restriction enzymes could be used to map and manipulate DNA 
[3]. After this, the field of molecular biology was truly born, leading to the synthesis of new drugs (e.g., insulin and growth hormone), gene mapping and sequencing, knock-out and knock-in models in mice, whole genome signaling, and much more. In fact, Arber, Smith, and Nathans were awarded the Nobel Prize in Physiology and Medicine in 1978, over 25 years after Luria's initial, seemingly trivial (descriptive) observation.

Another example comes from Paul Lauterbur, a physical chemist at Stonybrook who used NMR and other techniques to study protein structure. Once, the story goes that while out with colleagues, Lauterbur remarked that his NMR technique could possibly be used to look at structures of whole cells or perhaps even simple animals. Scribbling his ideas down on a napkin, Lauterbur went home and set to work, finally submitting a paper to Nature, where he used his strategy of "zeugmatography" to show the difference between heavy and ordinary water. It was a battle, but his manuscript was finally published [4]. The manuscript was esoteric - it would be hard for anybody to predict any translational potential from this dry description. However, Lauterbur was able to see beyond the horizon of his published application and proposed that a similar strategy could be used to see inside of a human, even going so far as to design a prototype machine to do so. Despite his efforts, neither the NIH nor even his own institution was willing to invest in this prototype, spouting concerns about the true translational value of what he was proposing to do. Perseverance prevailed, however, and several years later, Lauterbur completed his first human NMR machine, using funds primarily from foundations and other private organizations. Of course, the rest is history, culminating with a Nobel Prize in Physiology and Medicine in 2003 for developing the first MRI. Would study sections have looked upon Lauterbur's leap from physical chemistry to human anatomy measurements differently in 2014? It seems doubtful, though not due to biased or unfair reviewers, but instead due to the simple fact that Lauterbur was proposing to do something relatively risky if not crazy, with simply too little data to convincingly suggest an immediate translational potential.

Indeed, much has been written about translation in recent years $[5,6]$. The definition has evolved (Fig. $1 ;[5,7]$ ) as we (researchers in academia) clamber to adopt translational guidelines and approaches aimed at impressing NIH study section panelists. With sliding NIH budgets, should perceived or predicted impact or translational potential guide the prioritization of research spending? Again, we assert a resounding NO!

Translation (T1) typically moves from bench to bedside, but can flow in either direction; clinical challenges often serve to focus basic research questions. The process generally requires a great deal of time and money and involves innumerable basic and clinical observations made by countless dedicated researchers and clinicians. Collaboration across continents and between academic and industrial sectors is often required to drive translation. However, the process is unpredictable at best and often subject to coincidence. When the pathway to translation is obvious, most agree that "applied" research has the potential for "high impact." Is the converse always true? That is, when the path to translation is somewhat unclear or hard to predict (e.g., Luria's discovery of restricting bacterial hosts), does this mean the research has/ will have "low impact"? Does the timeline required to realize the clinical impact of a given novel discovery dampen that impact? Consider the over 40-year history of tamoxifen, a drug first developed as a potential (failed) contraceptive that is now the most widely used targeted therapy in medical oncology [8]. The story originated in 1896, with a seminal clinical observation made by pioneering breast cancer surgeon Dr. George Beatson, who discovered that surgical removal of the ovaries could extend the lives of some women with breast cancer [9]. Removal of the ovaries and adrenal glands became the standard of care "anti-hormone" approach for many decades (well into the 1970s). For unknown reasons, this treatment worked in only one of three patients.

During the 1950s, Dr. Elwood Jensen joined a team of researchers at the University of Chicago led by the renowned hormones and prostate cancer researcher Dr. Charles Huggins (1966 Nobel Laureate in Physiology or Medicine). Dr. Jensen's research focused on the hormone estrogen. He was the first to label the hormone estradiol with the radioactive isotope tritium to allow for sensitive detection in tissues [10]. His work led to the early discovery that approximately one third of all breast cancer patients had estrogen receptors (ERs) that bound to estrogen in their tumors leading to cell proliferation and provided an explanation (in part) for why roughly one third of patients responded to hormone ablative surgery (as noted above). Dr. Jensen was the first to purify the ER (alpha) and make specific antibodies to it [11], paving the way for routine clinical detection of ER in breast tumors. Today, it is recognized that about $70 \%$ of breast cancer patients have ER+ tumors (as defined by standardized clinical scoring criteria), but about $30 \%$ fail to respond to anti-estrogen therapy [12].

Meanwhile, in the 1960s, researchers in the UK led by reproductive endocrinologists Drs. Arthur Walpole and Michael Harper at ICI Pharmaceuticals (now AstraZeneca) were investigating estrogen-like compounds as potential regulators of blood lipids and for a variety of reproductive conditions, including as contraceptive (morning after pill) agents; the nonsteroidal anti-estrogen drug ICI46,474 was first synthesized by synthetic organic chemist Dora Richardson. The drug was not patent-protected in the USA and company support waned when "weak" or inconsistent (across species) contraceptive effects became apparent (cancer treatments were not an ICI corporate priority at that time). Following a few early cancer clinical trails conducted in Europe [13, 14], Walpole 


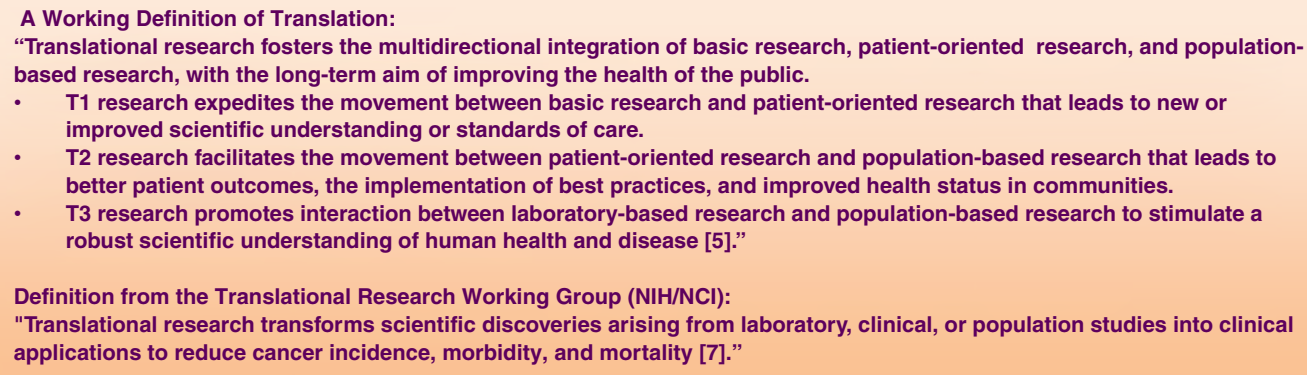

Fig. 1 Definitions of translation

convinced ICI to market the drug for late-stage breast cancer. ICI46,474 was developed into tamoxifen and doctors started giving it to breast cancer patients in the early 1970s; the drug was licensed for the treatment of advanced breast cancer in the UK (1973) and the USA (1977).

Progress was slow, as clinical enthusiasm for the use of drugs like tamoxifen to treat breast cancer patients remained sluggish. With the support of Dr. Walpole (and financially backed by ICI), Dr. V. Craig Jordan (and others [15]) took on the project of anti-estrogen action in breast cancer. Lippman and Bolan demonstrated the effects of estrogens/antiestrogens in cultured human breast cancer models in vitro [15]. Similarly, Jordan and Allen showed (Elwood Jensen had purified ER and developed the ER assay by this time) that tamoxifen worked well in the DMBA-induced rat model of mammary cancer [16]. Dr. Pierre Chambon's group cloned the ER (alpha) gene in 1986 [17]. These and many other basic studies predicted translation of similar results in women with ER + breast tumors [18]. The demonstration that prolonged treatment was better than transient treatment was also significant in the development of tamoxifen as a useful therapeutic agent [19]. In 1980, Baum and colleagues first reported that tamoxifen given in addition to chemotherapy improved survival for patients with early breast cancer [20]. Following a large body of related basic and clinical studies, the Early Breast Cancer Trialists' Collaborative Group showed (in a 1998 meta-analysis) definitively that tamoxifen saved lives in early breast cancer [21]. More recent studies continue to support this finding [22]. Prior to tamoxifen, the only way to target ER was to surgically remove the ovaries, adrenal glands, or pituitary gland. At the very least, taking tamoxifen provided a means of medically treating ER-expressing breast cancers without surgical intervention. Today, tamoxifen (the first in a class of related SERMs) and the class of drugs that followed as blockers of estrogen synthesis (aromatase inhibitors) have safely extended the lives of millions with breast cancer [8]. Anti-estrogen therapy is also approved for breast cancer prevention in high-risk groups [23, 24].

Is $40+$ years for translation of basic findings into clinical application too long? Would current standards consider the translational potential of Jensen's, Walpole's, Lippman's, and Jordan's early work too low? Perhaps, this can best be answered with another example. Similar to the circuitous path of tamoxifen (a "repurposed" contraceptive drug dating back to the 1960s), the benefits of trastuzumab/Herceptin ${ }^{\mathrm{TM}}$, the second major targeted therapy (to Her2/neu/erbB2 receptors) to emerge from the somewhat more focused world of translational breast cancer research, took at least 30 years to realize, from the basic science discovery of the first receptor tyrosine kinase (epidermal growth factor receptor) in 1978 [25] to the FDA approval (2008) of Herceptin ${ }^{\mathrm{TM}}$ (Genentech) in combination with chemotherapy for the adjuvant treatment of HER2-overexpressing node-negative (ER/PR-negative or with one high-risk feature) or node-positive breast cancer following multi-modality anthracycline-based therapy (reviewed in [26]). Today, Herceptin ${ }^{\mathrm{TM}}$, in combination chemotherapy and endocrine therapy in both advanced and operable breast cancer, extends lives [27-29]. Is 30 years still too long? Of note, only 11 years passed from the first report of evidence of Her2 amplification in breast cancer [30] to approval of Herceptin ${ }^{\mathrm{TM}}$ for metastatic disease (reviewed in [31]). Thus, when the basic science "leg-work" is mostly in place, can we accept the timeline of at least a decade? Let us ask the cancer survivors and their families, their doctors and nurses, the clinical and basic scientists who drive translation, their professional research staff, and the dedicated laboratory and animal facility technicians who support this work. Given that "hindsight is twenty-twenty," most of us would have supported even the longest of timelines from the above examples. Perhaps, we must simply acknowledge that it is virtually impossible to accurately assign scientific priorities when we cannot accurately predict the future.

Herein, we have provided just a handful of many examples of wildly successful research ventures that took decades of hard work by multiple investigators before any true translational results were generated. Our comments honor the need for basic science as the "seeds" for future innovation and impact in the form of translation. But, such stories bring forth a myriad of questions: Are we able to truly recognize impact or potential for translation? If we think that the answer is yes, 
can we shorten the timelines to translation? Should we even try? If so, how will we know when or if innovation has suffered? One cannot have a forest without thousands of trees, and each very important tree takes time and nurturing to grow. Where we as a scientific community draw the line between "basic" and translational, how we define "potential" must be carefully considered, and we must be cognizant of our limited ability to truly predict what will be ground breaking and relevant. To quote Arthur Schopenhauer from his "Studies in Pessimism: The Essays": "Every man takes the limits of his own field of vision for the limits of the world."

In closing, we remind our readers of the need to be better ambassadors for our science. We must continue to work harder than ever to improve the funding climate. The examples given above clearly show how science improves the lives of people. We need to continue to recruit advocates from the lay public, lobby our elected officials to improve cancer research funding, and support candidates who value and understand basic science.

Acknowledgments We thank Dr. Andrea Daniel (University of MN) for the technical editing assistance and Dr. Douglas Yee (University of $\mathrm{MN}$ ) for the helpful historical comments.

\section{References}

1. Roberts RJ (2005) How restriction enzymes became the workhorses of molecular biology. Proc Natl Acad Sci U S A 102(17):5905-5908

2. Smith H, Wilcox KW (1970) "A restriction enzyme from Hemophilus influenza 1. Purification and general properties," J Mol Biol 51:379-391

3. Raju TN (1999) The Nobel chronicles. 1978: Werner Arber (b 1929); Hamilton O Smith (b 1931); Daniel Nathans (b 1928). Lancet 354(9189):1567

4. Lauterbur P (1973) "Image formation by induced local interactions: examples employing nuclear magnetic resonance". Nature 242:190191

5. Rubio DM et al (2010) Defining translational research: implications for training. Acad Med 85(3):470-475

6. Vinson CA (2014) Using concept mapping to develop a conceptual framework for creating virtual communities of practice to translate cancer research into practice. Prev Chronic Dis 11:E68

7. NCI, T.R.W.G.

8. Jordan VC (2003) Tamoxifen: a most unlikely pioneering medicine. Nat Rev Drug Discov 2(3):205-213

9. Beatson GT (1896) On the treatment of inoperable cases of carcinoma of the mamma: suggestions for a new method of treatment with illustrative cases. Lancet 2:104-107

10. Jensen EV, Jacobson HI (1962) Basic guides to the mechanism of estrogen action. Recent Prog Horm Res 18:387-414

11. Greene GL et al (1977) Antibodies to estrogen receptor: immunochemical similarity of estrophilin from various mammalian species. Proc Natl Acad Sci U S A 74(9):3681-3685
12. Allred DC, Brown P, Medina D (2004) The origins of estrogen receptor alpha-positive and estrogen receptor alpha-negative human breast cancer. Breast Cancer Res 6(6):240-245

13. Cole MP, Jones CT, Todd ID (1971) A new anti-oestrogenic agent in late breast cancer. An early clinical appraisal of ICI46474. Br J Cancer 25(2):270-275

14. Ward HW (1973) Anti-oestrogen therapy for breast cancer: a trial of tamoxifen at two dose levels. Br Med J 1(5844):13-14

15. Lippman ME, Bolan G (1975) Oestrogen-responsive human breast cancer in long term tissue culture. Nature 256(5518): 592-593

16. Jordan VC, Allen KE (1980) Evaluation of the antitumour activity of the non-steroidal antioestrogen monohydroxytamoxifen in the DMBA-induced rat mammary carcinoma model. Eur J Cancer 16(2):239-251

17. Green $\mathrm{S}$ et al (1986) Cloning of the human oestrogen receptor cDNA. J Steroid Biochem 24(1):77-83

18. Cuzick J, Baum M (1985) Tamoxifen and contralateral breast cancer. Lancet 2(8449):282

19. Jordan VC, Allen KE, Dix CJ (1980) Pharmacology of tamoxifen in laboratory animals. Cancer Treat Rep 64(6-7):745-59

20. Baum $M$ et al (1983) Improved survival among patients treated with adjuvant tamoxifen after mastectomy for early breast cancer. Lancet 2(8347): 450

21. The Early Breast Cancer Clinical Trialists' Collaborative Group (1998) Tamoxifen for early breast cancer: an overview of the randomised trials. Early Breast Cancer Trialists' Collaborative Group. Lancet 351(9114):1451-67

22. Davies C et al (2011) Relevance of breast cancer hormone receptors and other factors to the efficacy of adjuvant tamoxifen: patient-level meta-analysis of randomised trials. Lancet 378(9793):771-784

23. Cuzick J et al (2007) Long-term results of tamoxifen prophylaxis for breast cancer - 96-month follow-up of the randomized IBIS-I trial. J Natl Cancer Inst 99(4):272-282

24. Powles TJ et al (2007) Twenty-year follow-up of the Royal Marsden randomized, double-blinded tamoxifen breast cancer prevention trial. J Natl Cancer Inst 99(4):283-290

25. Carpenter G, King L Jr, Cohen S (1978) Epidermal growth factor stimulates phosphorylation in membrane preparations in vitro. Nature. 276:409-410

26. Kumar GL, Badve SS (2008) Milestones in the Discovery of HER2 Proto-Oncogene and Trastuzumab (Herceptin ${ }^{\mathrm{TM}}$ )

27. Blackwell KL et al (2010) Randomized study of Lapatinib alone or in combination with trastuzumab in women with ErbB2-positive, trastuzumab-refractory metastatic breast cancer. J Clin Oncol 28(7): $1124-1130$

28. Viani GA et al (2007) Adjuvant trastuzumab in the treatment of her2-positive early breast cancer: a meta-analysis of published randomized trials. BMC Cancer 7:153

29. O’Shaughnessy J, Blackwell KL, Burstein H, Storniolo AM, Sledge G, Baselga J, Koehler M, Laabs S, Florance A, Roychowdhury D (2008) A randomized study of lapatinib alone or in combination with trastuzumab in heavily pretreated HER2 + metastatic breast cancer progressing on trastuzumab therapy. J Clin Oncol 26:1015, No 15S (May 20 Supplement

30. Slamon DJ et al (1987) Human breast cancer: correlation of relapse and survival with amplification of the HER-2/neu oncogene. Science 235(4785):177-182

31. Hudis CA (2007) Trastuzumab-mechanism of action and use in clinical practice. N Engl J Med 357(1):39-51 